\title{
A Simple Microstrip Bandstop Filter Using Cross-Coupling Stubs
}

\author{
Leung Chiu and Quan Xue \\ Department of Electronic Engineering, City University of Hong Kong, Hong Kong \\ Correspondence should be addressed to Leung Chiu, eechiuleung@yahoo.com.hk
}

Received 2 March 2012; Revised 26 June 2012; Accepted 10 July 2012

Academic Editor: Samir Trabelsi

Copyright ( 2012 L. Chiu and Q. Xue. This is an open access article distributed under the Creative Commons Attribution License, which permits unrestricted use, distribution, and reproduction in any medium, provided the original work is properly cited.

\begin{abstract}
This paper presents an alterative implementation of a shunt open-circuited stub for multilayer microwave circuit. With making use of the proposed implementation, a simple and compact bandstop filter with a district bandstop characteristics and a first spurious at the third harmonic is proposed. The proposed filter exhibits about $12.5 \%$ fractional bandwidth of $-10 \mathrm{~dB}$ signal rejection at the center frequency of $2.04 \mathrm{GHz}$ and flat group delay at the pass bands. Besides, by cascading a number of the proposed bandstop filters designed at the different frequencies, multiband bandstop filters can be easily realized. A dual- (tri-) band design at the center frequencies of $2 \mathrm{GHz}$ and $3 \mathrm{GHz}$ (and $4 \mathrm{GHz}$ ) is designed, realized, and measured. The proposed bandstop filter well suits the nowadays multilayer and compact radio frequency integrated circuit design.
\end{abstract}

\section{Introduction}

Bandstop filter is one of the indispensable components for radio frequency and microwave circuits' applications. This filter rejects unwanted signals such as harmonic and spurious signals and allows desired signal to pass through. Recently, a kind of bandstop filter has been integrated into nonlinear circuits such as mixer, oscillator, and amplifier to enhance their performances [1-3].

Electromagnetic band gap (EBG) and resonant structures are found interesting in designing bandstop filter. EBG structure exhibits bandstop effect because of the band gap effect $[4,5]$. EBG structure is a periodic structure; hence, the strength of the bandstop effectiveness is determined by the number of stage of the unit cells. It occupies large circuit area if a strong or a significant bandstop effect is required.

Patterned ground structures and resonance cells belong to the family of the resonant structures. They provide bandstop effect by its nature resonance characteristics. For the patterned ground structure, the slotted pattern on the ground plane can be modeled as an $L C$ resonator to have strong bandstop effect; however, strong radiation is found on the pattern ground to introduce electromagnetic compatibility and interference issues. Besides, the reported works on the resonance cells cannot achieve low insertion losses at the both lower and higher pass bands at the same time leading to the limited applications [6].

A shunt open-circuited stub is the simplest bandstop structure for microwave circuit design. Figure 1 shows the schematic diagram of the proposed bandstop structure modified from a simple shunt open-circuited stub and its frequency response. It exhibits distinct bandstop characteristics with sharp roll-off and the first spurious response at the third harmonic. It consists of a pair of quarter wavelength opencircuited stubs separated by quarter wavelength, where $Z_{0}$ is the port impedance, $Z_{S}$ is the characteristics impedance of the shunt stub, and $C$ is capacitance between the two stubs. Cross-coupling between the two stubs helps to enhance the bandstop effect by introducing two transmission zeros within the stop band. A bandstop filter with similar schematic diagram was reported in [7]. The idea is realized by planar microstrip lines; however, it is still found that it occupies large circuit area.

In this paper, a multilayer structure to realize the modified bandstop structure as shown in Figure 1(a) is proposed. Recently, low-temperature cofire ceramic and CMOS techniques are found particularly suitable to realize multilayer structure to further reduce the circuit size. The proposed concept provides an alternative solution for the integrated circuit to realize shunt open-circuited stubs and other related circuit elements. 


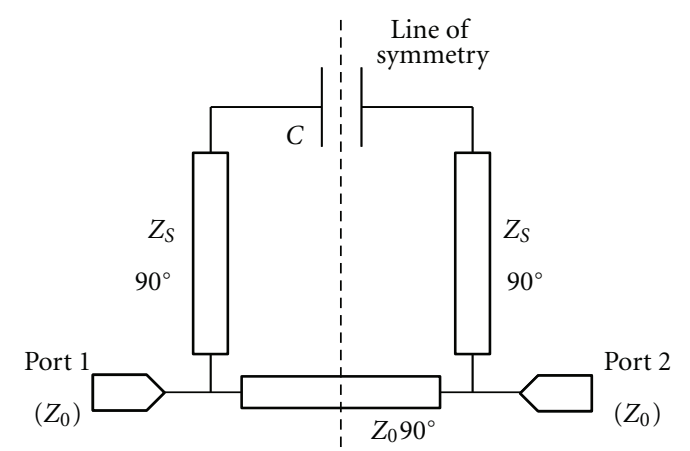

(a)
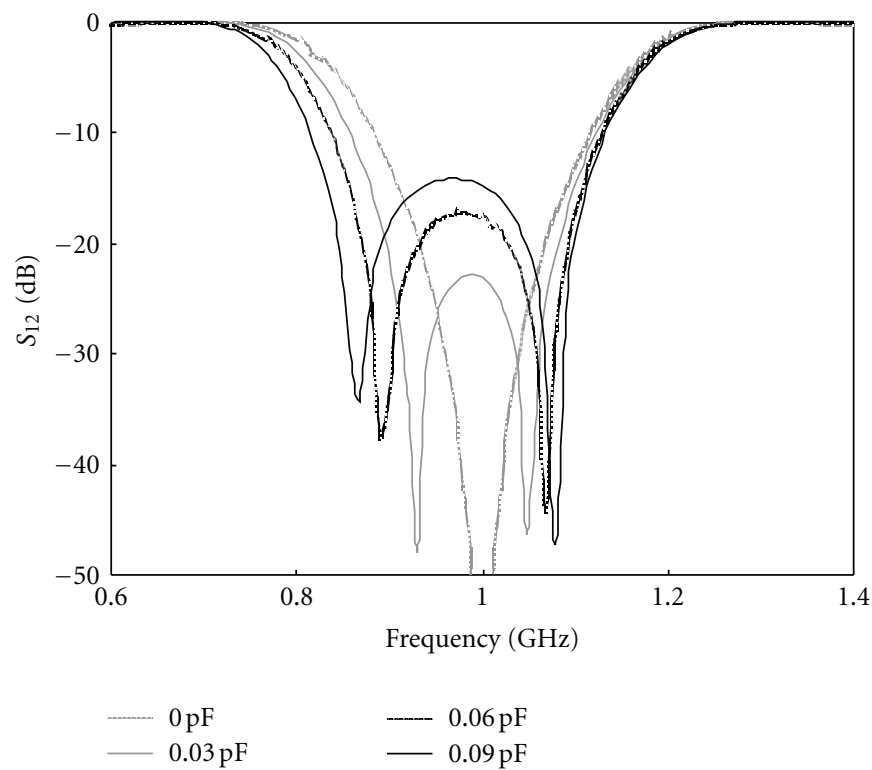

(b)

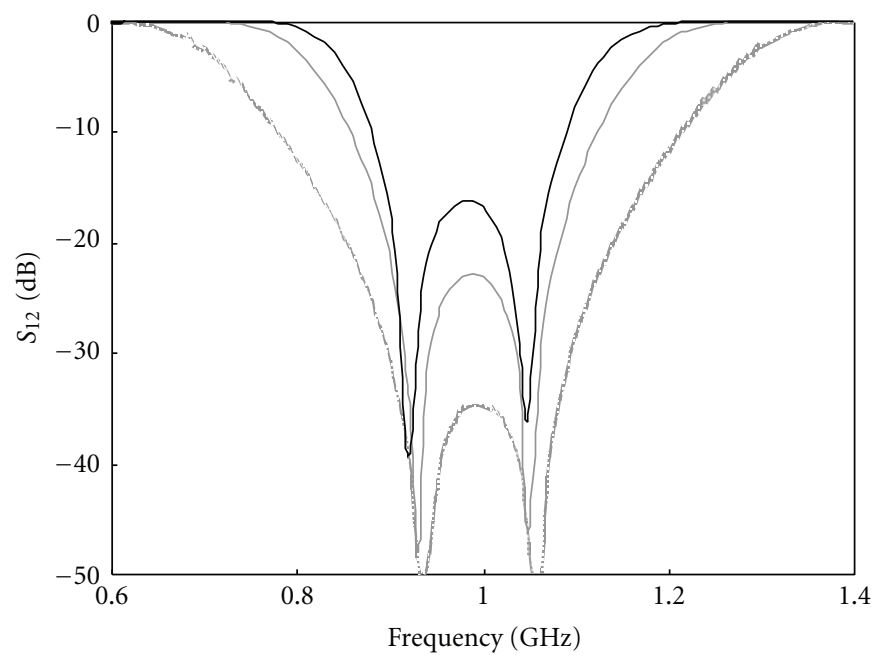

$50 \Omega$
$-100 \Omega$
$-150 \Omega$

(c)

Figure 1: (a) Schematic diagram of the proposed bandstop filter. (b) Calculated frequency response of the $\left|S_{21}\right|$ with different $C$ and fixed $Z_{S}=100 \Omega$. (c) Calculated frequency response of the $\left|S_{21}\right|$ with different $Z_{S}$ and fixed $C=0.03 \mathrm{pF}$. 


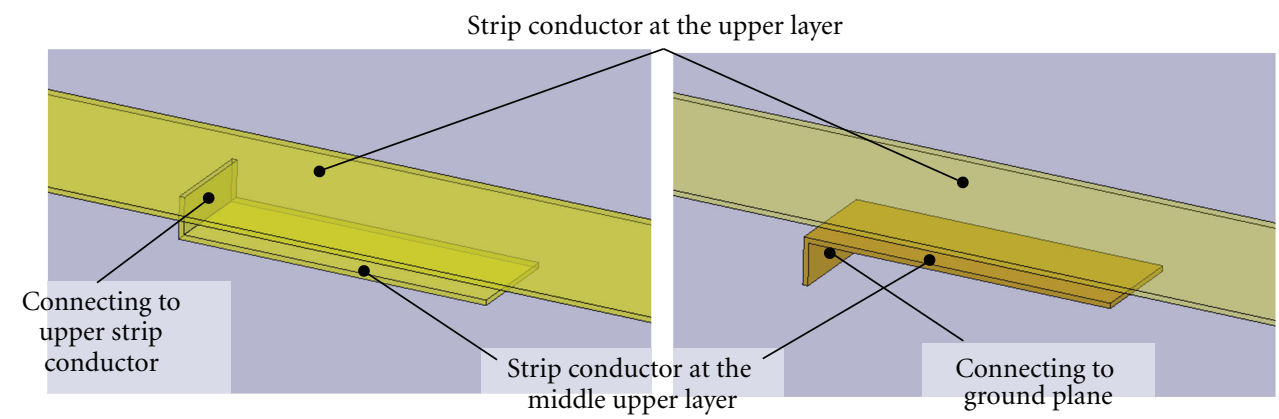

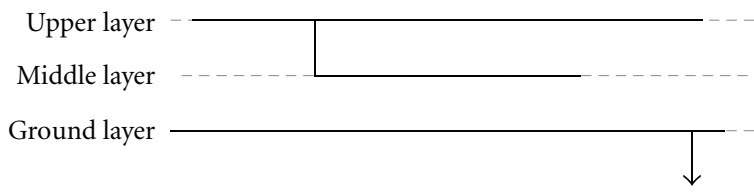

(a) impedances of the two shunt stubs and the section of transmission line are $Z_{S}$ and $Z_{0}$, respectively. The electrical lengths of all lines are $90^{\circ}$. The cross-coupling is modeled by the capacitor $C$ connecting between the ends of the two stubs.

With the symmetric structure of the proposed filter, even- and odd-mode analysis can be applied. The $S$ parameters of the structure shown in Figure 1(a) are given by

$$
\begin{aligned}
& S_{11}=S_{22}=\frac{1}{2}\left\{\frac{1-j\left(\left(Z_{0} / Z_{S}\right) \tan 2 \theta+\tan \theta\right)}{1+j\left(\left(Z_{0} / Z_{S}\right) \tan 2 \theta+\tan \theta\right)}\right. \\
& \left.+\frac{1+j\left[\left(Z_{0} / Z_{S}\right)\left(\left(2 \omega C Z_{S}+\tan 2 \theta\right) /\left(2 \omega C Z_{S} \tan 2 \theta-1\right)\right)+(1 / \tan \theta)\right]}{1-j\left[\left(Z_{0} / Z_{S}\right)\left(\left(2 \omega C Z_{S}+\tan 2 \theta\right) /\left(2 \omega C Z_{S} \tan 2 \theta-1\right)\right)+(1 / \tan \theta)\right]}\right\} \\
& S_{12}=S_{21}=\frac{1}{2}\left\{\frac{1-j\left(\left(Z_{0} / Z_{S}\right) \tan 2 \theta+\tan \theta\right)}{1+j\left(\left(Z_{0} / Z_{S}\right) \tan 2 \theta+\tan \theta\right)}\right. \\
& \left.-\frac{1+j\left[\left(Z_{0} / Z_{S}\right)\left(\left(2 \omega C Z_{S}+\tan 2 \theta\right) /\left(2 \omega C Z_{S} \tan 2 \theta-1\right)\right)+(1 / \tan \theta)\right]}{1-j\left[\left(Z_{0} / Z_{S}\right)\left(\left(2 \omega C Z_{S}+\tan 2 \theta\right) /\left(2 \omega C Z_{S} \tan 2 \theta-1\right)\right)+(1 / \tan \theta)\right]}\right\},
\end{aligned}
$$

where $\theta=\left(\pi f / 4 f_{0}\right) . f$ and $f_{0}$ are the center frequency and operating frequency, respectively. $Z_{0}=50 \Omega$ is chosen and is the same as port impedance. Parametric studies with different values of $C$ and $Z_{S}$ are shown in Figures 1(b) and $1(\mathrm{c})$, respectively. The cross-coupling introduces two transmission zeros at the stop band. The larger $C$ results in the stronger cross-coupling between the two stubs. The stop band bandwidth and roll-off can be improved by introducing suitable cross-coupling. With keeping the same $C$, the stop band bandwidth is adjusted by the characteristic impedance $Z_{S}$ of the stubs. The bandwidth is reduced with increasing $Z_{S}$.

\section{Multilayer Shunt Open-Circuited Stub Implementation}

Figure 2 shows the geometry of the proposed implementation of microstrip shunt open-circuited stub. It consists of three conductor layers, namely, ground plane, middle strip layer, and upper strip layer. Dielectric substrate is completely filled between the layers. The dielectric substrate used has dielectric constant of 2.65, substrate thickness of each dielectric layer of $0.8 \mathrm{~mm}$, and metal thickness of $0.02 \mathrm{~mm}$. The thicknesses of the two dielectric substrates are 


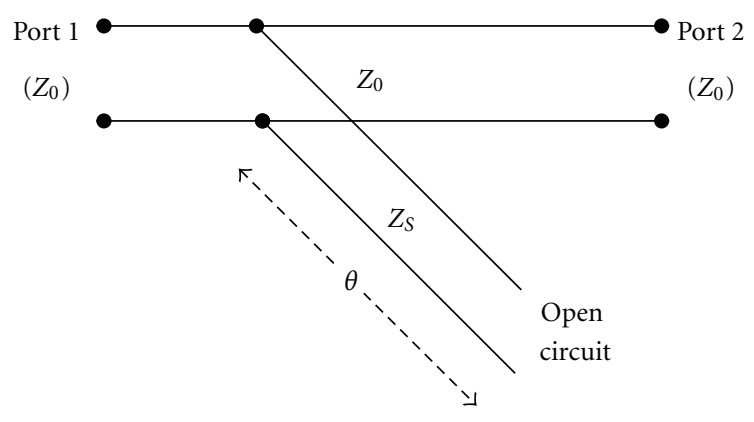

(a)

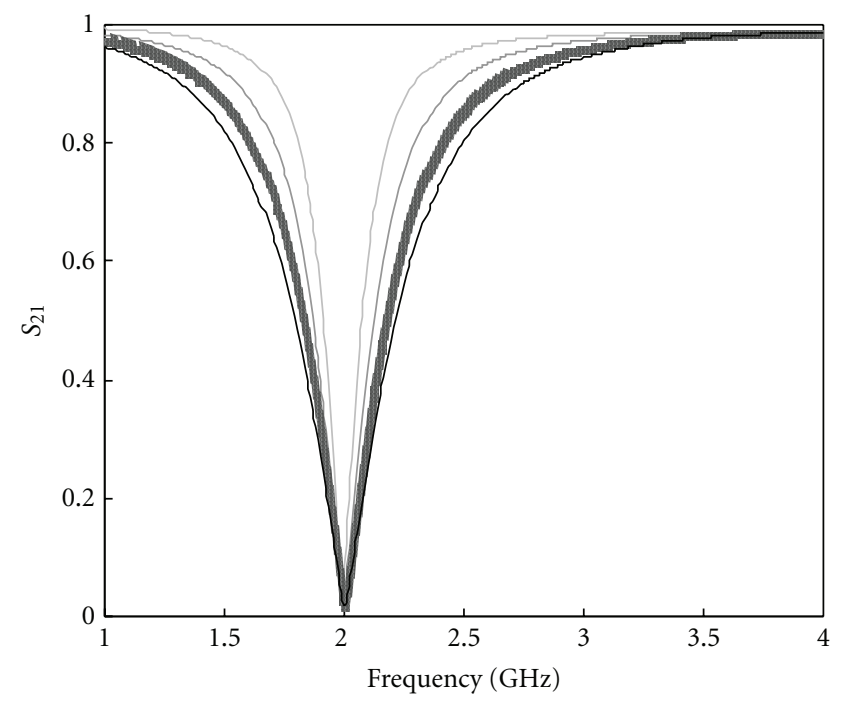

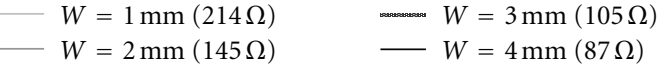

(b)

FIGURE 3: (a) The equivalent circuit model of the structure as shown in Figure 2(b). (b) Simulated frequency responses of $\left|S_{21}\right|$ of the structure as shown in Figure 2(b) with different width at middle layer and the corresponding equivalent $Z_{S}$, where $\theta$ is set to be $90^{\circ}$ at $2 \mathrm{GHz}$.

not necessarily equal, while these thicknesses are assigned to be equal in this paper.

The proposed implementation has no difference from the conventional microstrip line, except that there are a middle conductor layer and a metallic via between middle and either upper or ground layer. The two different versions of the implementations as shown in Figures 2(a) and 2(b) are exactly the same theoretically. It is preferable to connect the middle and ground layers by the metallic via as shown in Figure 2(b), since it can have smaller insertion loss and is easier to integrate lumped element or active device on the upper layer than that as shown in Figure 2(a) in the practical implementation.

Figure 3(a) shows the equivalent circuit model of the structure as shown in Figure 2(b). The length of the conductor at the middle is set to be quarter wavelength. By tuning its width, the characteristics impedance of shunt stub in the equivalent circuit can be changed. Figure 3

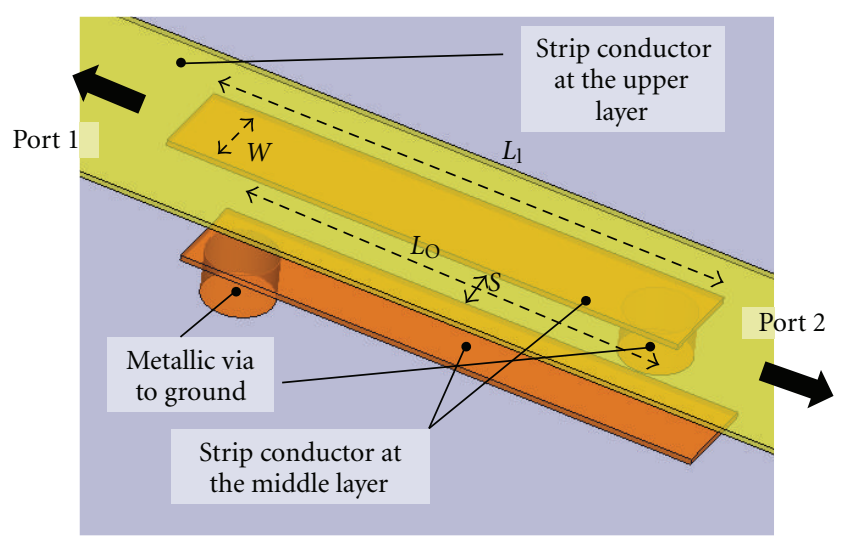

(a)

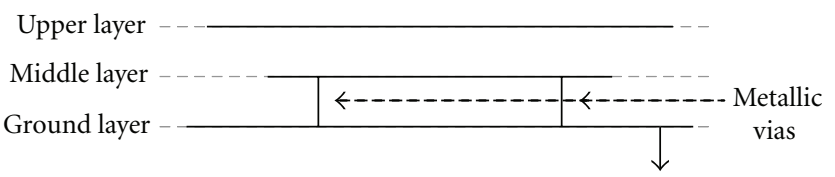

(b)

Figure 4: 3D and side views of the proposed bandstop filter using the proposed implementation with dimensions of the line width ( $W=1.5 \mathrm{~mm})$, the line length $\left(L_{l}=23 \mathrm{~mm}\right)$, the line separation $(S=1.44 \mathrm{~mm})$, and the overlapping line $\left(L_{O}=20.2 \mathrm{~mm}\right)$.

shows the bandstop characteristics with different conductor width at the middle layer. The parametric study showing the relationship between the conductor width and the corresponding characteristics impedance $\left(Z_{S}\right)$ is shown in Figure 3(b).

\section{Application: Bandstop Filter}

4.1. Single-Band Design. A working prototype was realized by a standard printed circuit board fabrication technique. The dielectric substrate used is the same as reported in previous section. By arranging two shunt open-circuited stubs in the antiparallel configuration as shown in Figure 4, a bandstop filter is realized. With keeping uniform width of a $50 \Omega$ line, we just tune the line width $(W=1.5 \mathrm{~mm})$, the line length $\left(L_{l}=23 \mathrm{~mm}\right)$, the line separation $(S=1.44 \mathrm{~mm})$, and the overlapping line $\left(L_{O}=20.2 \mathrm{~mm}\right)$. The entire printed circuit board size is $10 \mathrm{~cm}$ by $5 \mathrm{~cm}$.

Figure 5 shows the both simulated and measured $S$ parameters and group delays of the proposed bandstop filter. The corresponding values of the circuit model as shown in Figure 1 (a) are $Z_{0}=50 \Omega, Z_{S}=209 \Omega$, and $C=3.55 \mathrm{fF}$, and the center frequency is set to be $2 \mathrm{GHz}$. It exhibits distinct stop band at the center frequency of $2.04 \mathrm{GHz}$ with the first spurious response at about $6 \mathrm{GHz}$. The measured $-10 \mathrm{~dB}$ signal rejection bandwidth is $12.5 \%$. Acceptable agreement between the model, the simulation, and the measurement is obtained. However, the soldering between the via and the conductors are not modeled resulting in the inaccurate prediction especially of the return loss performance as shown in Figure 5(b). 


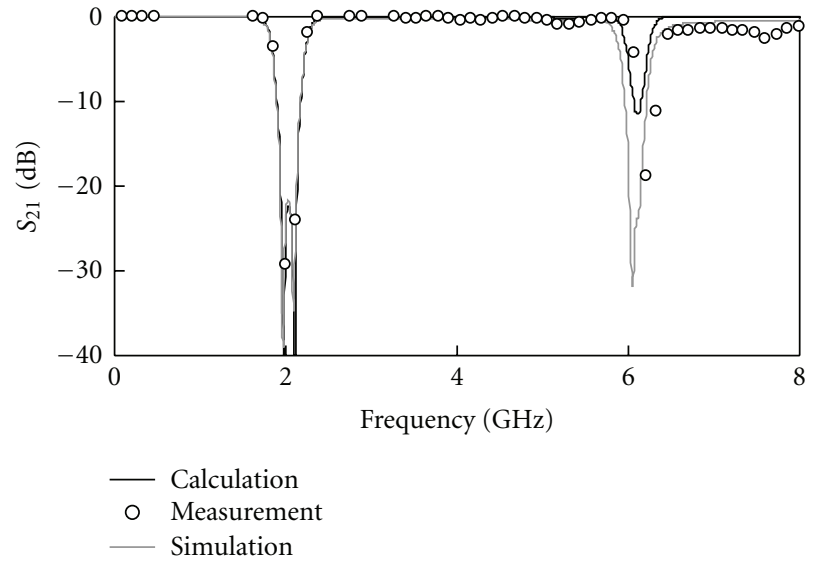

(a)

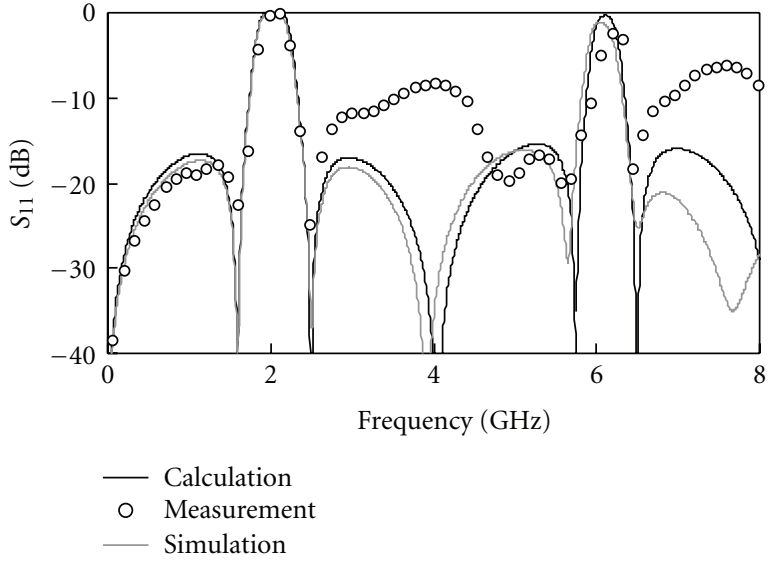

(b)

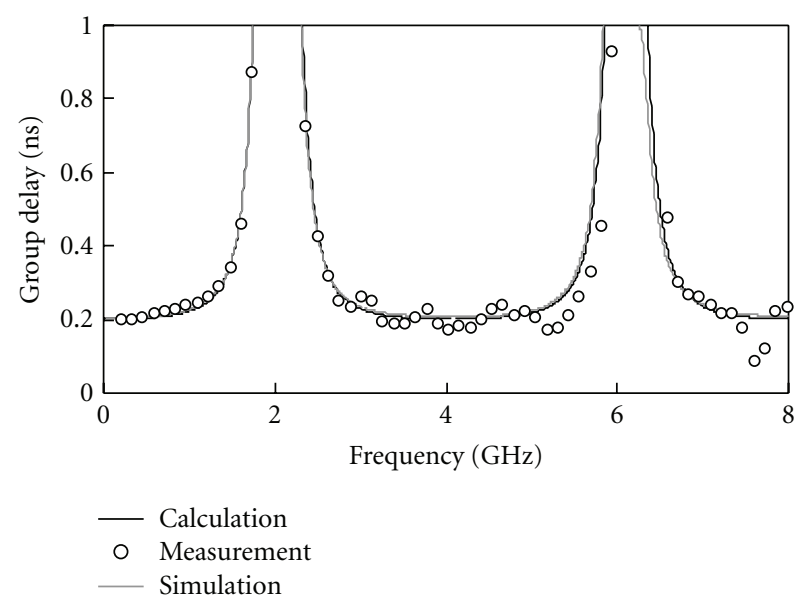

(c)

FIGURE 5: Calculated, simulated, and measured performances of the proposed single-bandstop filter. (a) $S_{21}$, (b) $S_{11}$, and (c) group delay.

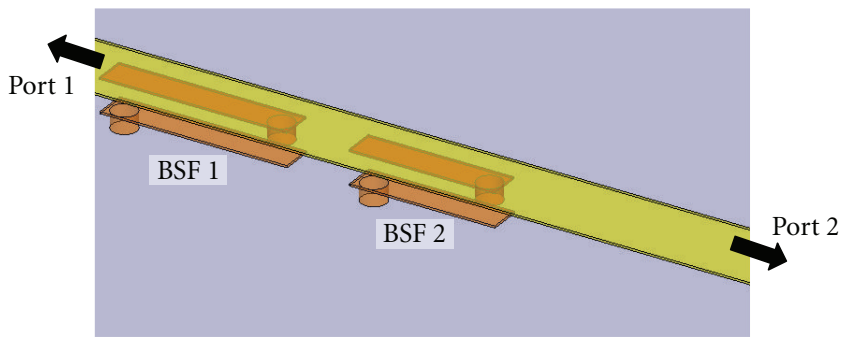

(a)

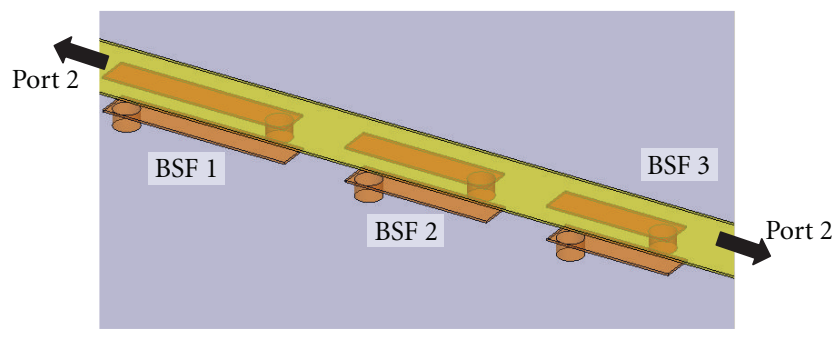

(b)

FIGURE 6: Geometries of the proposed multiband bandstop filters consisting a number of subbandstop filters (BSFs). (a) Dual-band bandstop filter. (b) Triband bandstop filter.

4.2. Multiband Design. In order to meet different standards simultaneously, dual-band or multiband designs should be employed to meet the various specializations. By connecting a number of the proposed bandstop filters designed at different frequencies in series connection as shown in Figure 6, a multiband design can easily be realized. A dualband design at the center frequencies of about $2 \mathrm{GHz}$ and
$3 \mathrm{GHz}$ and a triband design at the center frequencies of about $2 \mathrm{GHz}, 3 \mathrm{GHz}$, and $4 \mathrm{GHz}$ are realized to demonstrate the multiband concept. Their simulated and measured performances are shown in Figure 7. The dual-band design offers two distinct stop bands at the center frequencies of $2.04 \mathrm{GHz}$ and $2.87 \mathrm{GHz}$ with fractional bandwidths of $12.5 \%$ and $11.8 \%$, respectively, while the triband design offers 


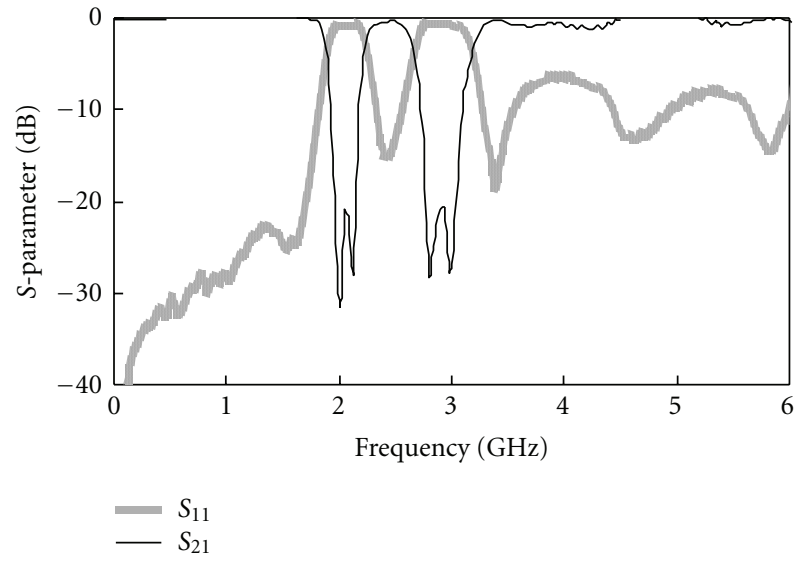

(a)

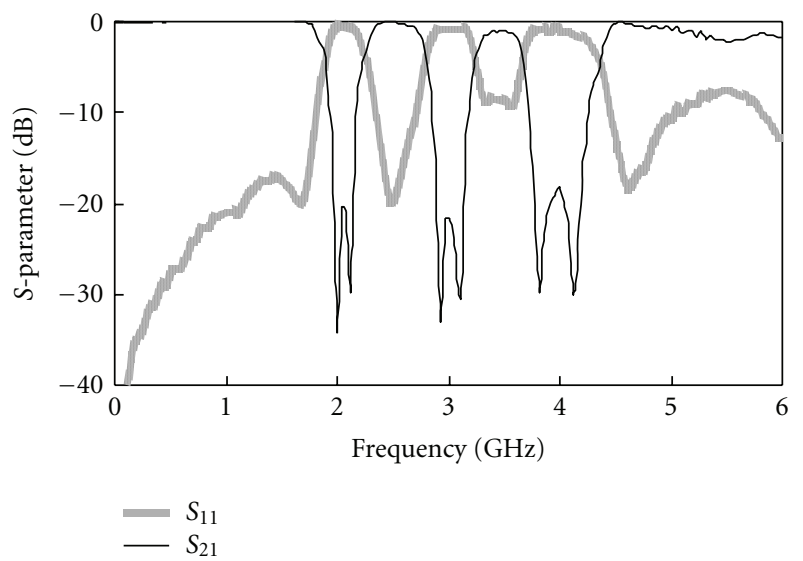

(b)

FIGURE 7: Measured S-parameters of the proposed multiband bandstop filters. (a) Dual-band bandstop filter. (b) Triband bandstop filter.

an extra stop band at the center frequencies of $3.97 \mathrm{GHz}$ with fractional bandwidths of $13.9 \%$. The dimension of each section of the multiband bandstop filter can be designed separately and each section is almost independent to others. The $3 \mathrm{GHz}$ bandstop filter (BSF2) has physical dimensions of $W=1.50 \mathrm{~mm}, L_{l}=16.0 \mathrm{~mm}, S=1.44 \mathrm{~mm}$, and $L_{O}=13.2 \mathrm{~mm}$ and electrical parameters of $Z_{0}=50 \Omega, Z_{S}=$ $209 \Omega$, and $C=2.37 \mathrm{fF}$. The $4 \mathrm{GHz}$ bandstop filter (BSF3) has physical dimensions of $W=1.50 \mathrm{~mm}, L_{l}=11.5 \mathrm{~mm}$, $S=1.44 \mathrm{~mm}$, and $L_{O}=8.70 \mathrm{~mm}$ and electrical parameters of $Z_{0}=50 \Omega, Z_{S}=209 \Omega$, and $C=1.78 \mathrm{fF}$. The two filters were built on the two separated printed circuit boards with physical size of $10 \mathrm{~cm}$ by $5 \mathrm{~cm}$. The proposed bandstop filters achieve very compact design without changing the signal line, while some of the previously proposed filters such as [5-7] achieve bandstop performance by etching 2D patterns on the single line or dielectric substrate.

\section{Conclusion}

A new shunt stub implementation scheme is proposed for bandstop filter design. Distinct bandstop ability with sharp roll-off is achieved by cross-coupling between two quarterwave shunt stubs separated by a section of transmission line with quarter wavelength long. A flat response of the group delay at the pass bands is achieved. By connecting several bandstop filters designed at the different frequencies, a multiband design can be easily realized. It is a simple bandstop structure, which well suits multilayer integrated circuit design. The proposed filter achieves 1D design, while the previous bandstop filter requires $2 \mathrm{D}$ patterns on the signal lines.

\section{Acknowledgments}

This work was supported in part by the Science and Technology Development Fund of Macau SAR under Grant 020/2009/A1 and by Shenzhen Science and Technology
Planning Project for the Establishment of Key Laboratory in 2009 (project CXB200903090021A).

\section{References}

[1] L. Chiu, T. Y. Yum, Q. Xue, and C. H. Chan, "A low-conversionloss ring-hybrid mixer using TS-CMRC circuitry," Microwave and Optical Technology Letters, vol. 41, no. 3, pp. 206-209, 2004.

[2] Q. Xue, K. M. Shum, and C. H. Chan, "Novel oscillator incorporating a compact microstrip resonant cell," IEEE Microwave and Wireless Components Letters, vol. 11, no. 5, pp. 202-204, 2001.

[3] T. Y. Yum, Q. Xue, and C. H. Chan, "Amplifier linearization using compact microstrip resonant cell-theory and experiment," IEEE Transactions on Microwave Theory and Techniques, vol. 52, no. 3, pp. 927-934, 2004.

[4] V. Radisic, Y. Qian, and T. Itoh, "Broad-band power amplifier using dielectric photonic bandgap structure," IEEE Microwave and Guided Wave Letters, vol. 8, no. 1, pp. 13-14, 1998.

[5] V. Radisic, Y. Qian, R. Coccioli, and T. Itoh, "Novel 2D photonic bandgap structure for microstrip lines," IEEE Microwave and Guided Wave Letters, vol. 8, no. 2, pp. 69-71, 1998.

[6] Q. Xue, K. M. Shum, and C. H. Chan, "Novel 1-D microstrip PBG cells," IEEE Microwave and Wireless Components Letters, vol. 10, no. 10, pp. 403-405, 2000.

[7] H. Shaman and J. S. Hong, "Wideband bandstop filter with cross-coupling," IEEE Transactions on Microwave Theory and Techniques, vol. 55, no. 8, pp. 1780-1785, 2007. 

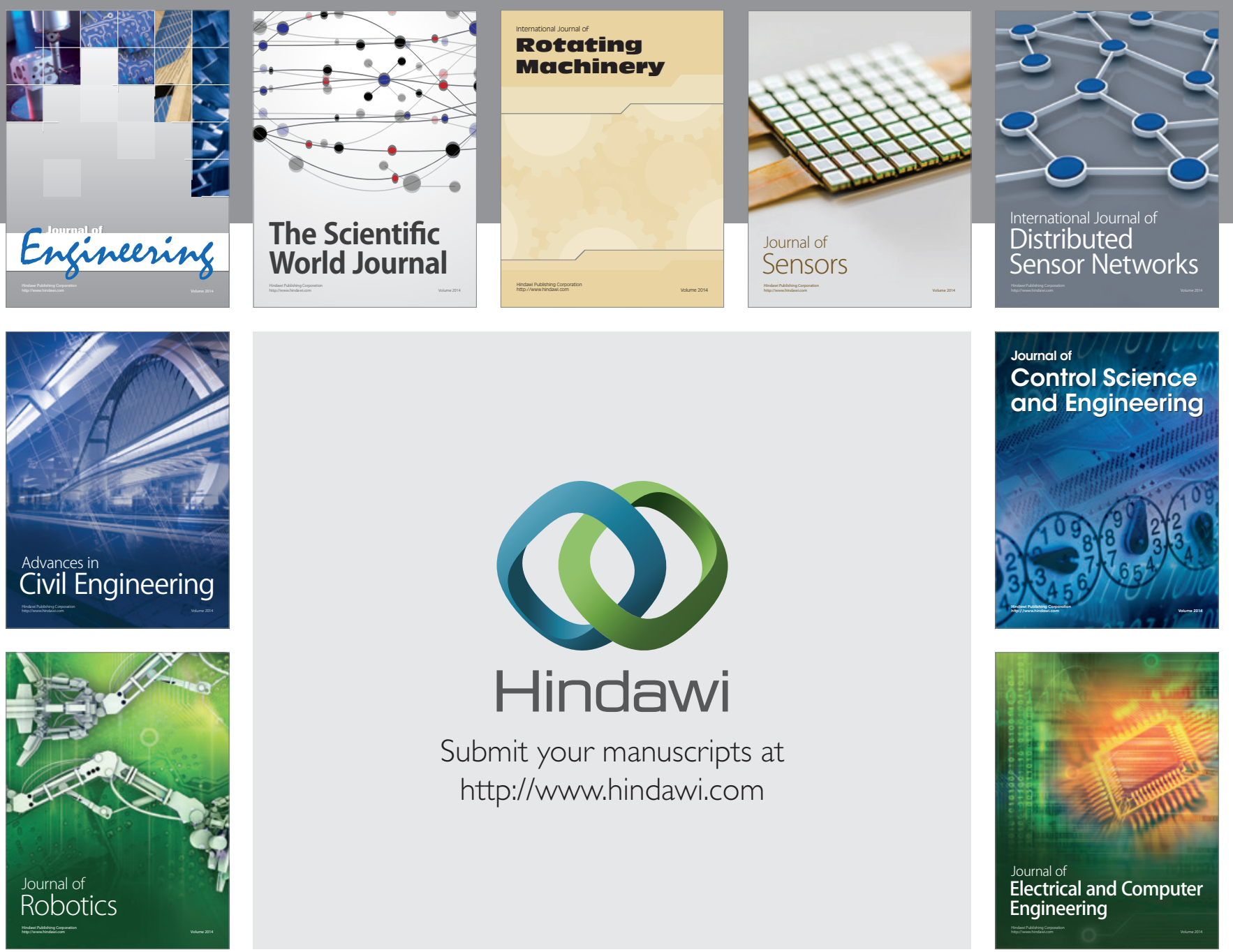

Submit your manuscripts at

http://www.hindawi.com
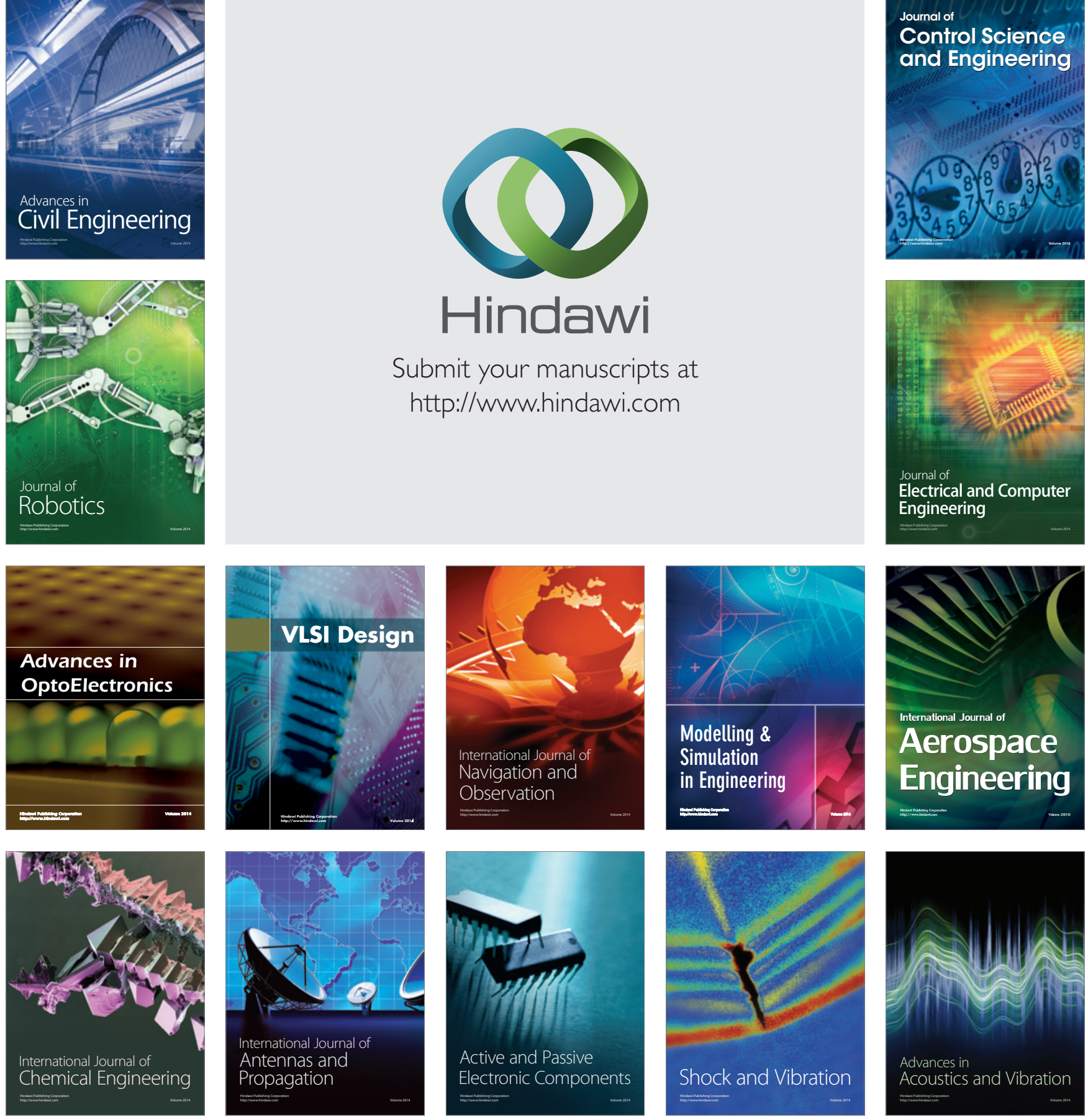